\title{
Sphere Packing Modulation in the SDMA Uplink Using $K$-Best Sphere Detection
}

\author{
Li Wang, Osamah Alamri, and Lajos Hanzo
}

\begin{abstract}
Recently, the turbo-detected sphere packing (SP) aided space-time block-coding (STBC) STBC-SP scheme was demonstrated to provide useful performance improvements over conventionally-modulated orthogonal design-based STBC schemes in the context of single-user multiple-input multiple-output (SU-MIMO) systems. Hence, in this letter, we extend its employment to the multiuser MIMO (MU-MIMO) scenario. For the sake of achieving a near maximum a posteriori (MAP) performance while imposing a moderate complexity, we specifically design the $K$-best sphere detection (SD) scheme for supporting the operation of the SP-modulated system, since the conventional SD cannot be directly applied to such a system. Consequently, with the aid of our modified SD, a significant performance gain can be achieved by the SP-modulated system over its conventionally-modulated counterpart in the context of MU-MIMO systems.
\end{abstract}

\section{INTRODUCTION}

$\mathbf{T}$ HE concept of combining orthogonal transmit diversity designs with the principle of sphere packing (SP) [1] was introduced by $\mathrm{Su}$ et al. in [2] in order to maximize the achievable coding advantage, demonstrating that the proposed SP-aided STBC (STBC-SP) scheme, was capable of outperforming the conventional orthogonal design-based STBC schemes of [3]-[5] in the single-user MIMO (SU-MIMO) scenario. In parallel, sphere detection (SD) techniques [6], [7] designed for conventionally-modulated MIMO systems have received wide interest in both the research and industrial communities, which are capable of achieving a near-MAP performance at a significantly lower computational complexity compared to the classic MAP detectors.

Against this background, we design a STBC-SP scheme for a MU-MIMO SDMA scenario with the aid of the SD technique. Our main contribution in this letter is the challenging design of the $K$-best SD for SP-modulated systems, which extends the employment of STBC-SP schemes to MU-MIMO scenarios, while approaching the MAP performance at a moderate complexity: 1) We improve the STBC performance by jointly designing the space-time signals of the two time slots of an SDMA scheme using SP modulation, while existing designs make no attempt to do so owing to its complex detection. 2) We solve this complexity problem by further developing the $K$-best SD for the detection of SP modulation, because SP offers a substantial SNR reduction at a potentially excessive complexity, which is reduced by the new SD.

Notation: The bold uppercase variables, such as $\mathbf{X}$, and the lowercase variables $\mathbf{x}$ denote matrices and vectors, respectively.

Manuscript received May 30, 2008; revised November 21, 2008. Current version published February 25, 2009. This work was supported in part by the Virtual Centre of Excellence in Mobile and Personal Communications, Mobile VCE and in part by EPSRC, U.K. The associate editor coordinating the review of this manuscript and approving it for publication was Prof. Martin Haardt.

The authors are with School of Electronics and Computer Science, University of Southampton, Southampton SO17 1BJ, U.K.

Digital Object Identifier 10.1109/LSP.2008.2012219

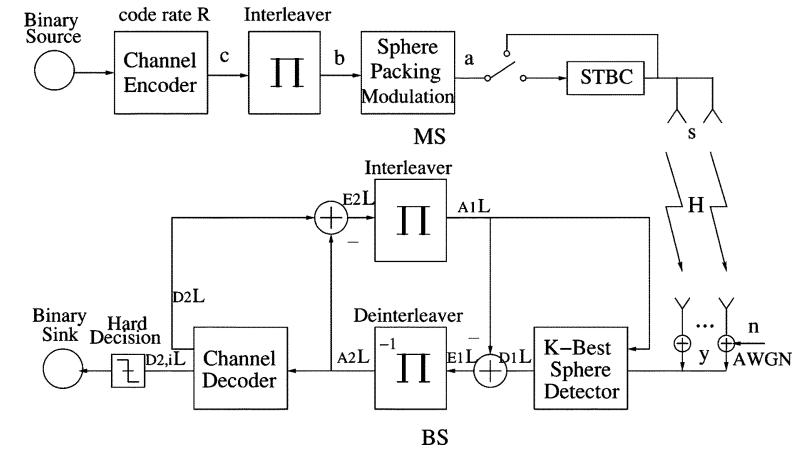

Fig. 1. Schematic of the uplink SP modulated multiuser MIMO system using $K$-best SD.

The element in the $i$ th row and $j$ th column of a matrix $\mathbf{X}$ is represented by $\mathbf{X}_{i, j}$, while the $i$ th element of a vector $\mathbf{x}$ is denoted by $\mathbf{x}_{i}$.

\section{SYSTEM OVERVIEW}

In this letter, we consider an STBC-aided uplink (UL) MU-MIMO scenario, where the spatial division multiple access (SDMA) [8] system supports $U$ UL users and employs $N$ receive antennas at the BS. For the sake of simplicity, the well-known STBC scheme proposed by Alamouti in [3], also known as the $\mathbf{G}_{2}$ scheme using two transmit antennas, is employed by each user. The schematic of the system is depicted in Fig. 1, where the transmitted source bits of the $u$ th user are channel encoded and then interleaved by a random bit-interleaver. The $B$ interleaved bits $\mathbf{b}^{(u)}=b_{0, \ldots, B-1}^{(u)} \in\{0,1\}$ are mapped to one of the $L=2^{B}$ legitimate four-dimensional SP symbols $\vec{a}^{(u)} \in \mathcal{A}$ by the SP modulator/mapper of Fig. 1, where $\mathcal{A}=\left\{\vec{a}_{l}=\left[a_{l, 1}, a_{l, 2}, a_{l, 3}, a_{l, 4}\right]^{T} \in D_{4} \subset\right.$ $\left.\mathbb{R}^{4}: 0 \leq l \leq L-1\right\}$ constitutes a set of $L$ constellation points from the entire set of legitimate SP constellation points hosted by the lattice $D_{4}$ [1] having a total energy of $E_{\text {total }} \triangleq \sum_{l=1}^{L}\left(\left|a_{l, 1}\right|^{2}+\left|a_{l, 2}\right|^{2}+\left|a_{l, 3}\right|^{2}+\left|a_{l, 4}\right|^{2}\right)$. The $\mathbf{G}_{2}$ encoder then maps the SP modulated symbol $\vec{a}^{(u)}$ to a space-time signal $\mathbf{C}^{(u)}=\sqrt{(2 L) /\left(E_{\text {total }}\right)} \mathbf{G}_{2}\left(F_{4}\left(\vec{a}^{(u)}\right)\right)[1]$, [2] by invoking the following two equations:

$$
F_{4}\left(a_{l, 1}, a_{l, 2}, a_{l, 3}, a_{l, 4}\right) \triangleq(\underbrace{a_{l, 1}+j a_{l, 2}}_{x_{1}}, \underbrace{a_{l, 3}+j a_{l, 4}}_{x_{2}})
$$

and

$$
\mathbf{G}_{2}\left(x_{1}, x_{2}\right)=\left[\begin{array}{cc}
x_{1} & -x_{2}^{*} \\
x_{2} & x_{1}^{*}
\end{array}\right]
$$

which are the $\mathbb{R}^{4} \rightarrow \mathbb{C}^{2}$ SP symbol transformation equation and the $\mathbf{G}_{2}$ signaling matrix [3], respectively. Finally, the spacetime signal $\mathbf{C}^{(u)}$ is transmitted from the two transmit antennas of the $u$ th MS during two consecutive time slots. 
In Fig. 1 the interleaver and deinterleaver pair seen at the BS divides the receiver into two parts, namely, the SD and the channel decoder. Note that in Fig. $1, L_{A}, L_{E}$, and $L_{D}$ denote the a priori, the extrinsic, and the a posteriori log-likelihood-ratios (LLRs), while the subscript "1" and "2" represent the bit LLRs associated with the SD and the channel decoder, respectively. It was detailed throughout [9] that the iterative exchange of extrinsic information between these serially concatenated receiver blocks results in substantial performance improvements. In this letter, we assume familiarity with the classic turbo detection principles [9].

Under the assumption that the channel impulse response (CIR) taps between each of the two transmit antennas of a specific user and the $n$th receive antenna at the BS remain constant during two consecutive symbol periods, the overall equivalent system equation of the MU-MIMO scenario of Fig. 1 can be derived as

$$
\begin{aligned}
\tilde{\mathbf{y}} & =\left[\begin{array}{c}
\tilde{\mathbf{y}}_{1} \\
\tilde{\mathbf{y}}_{2} \\
\vdots \\
\tilde{\mathbf{y}}_{N}
\end{array}\right]=\left[\begin{array}{cc}
\sum_{u=1}^{U} \tilde{\mathbf{H}}_{1}^{(u)} \cdot \mathbf{x}^{(u)} \\
\sum_{u=1}^{U} \tilde{\mathbf{H}}_{2}^{(u)} \cdot \mathbf{x}^{(u)} \\
\vdots \\
\sum_{u=1}^{U} & \tilde{\mathbf{H}}_{N}^{(u)} \cdot \mathbf{x}^{(u)}
\end{array}\right]+\left[\begin{array}{c}
\tilde{\mathbf{w}}_{1} \\
\tilde{\mathbf{w}}_{2} \\
\vdots \\
\tilde{\mathbf{w}}_{N}
\end{array}\right] \\
& =\tilde{\mathbf{H}} \cdot \mathbf{x}+\tilde{\mathbf{w}}
\end{aligned}
$$

where upon defining

$$
\tilde{\mathbf{H}}_{n}^{(u)}=\left[\begin{array}{cc}
h_{n 1}^{(u)} & h_{n 2}^{(u)} \\
h_{n 2}^{(u) *} & -h_{n 1}^{(u) *}
\end{array}\right]
$$

with $h_{n 1}^{(u)}$ and $h_{n 2}^{(u)}$ representing the UL CIR coefficients between the first and second transmit antennas of the $u$ th user to the $n$th BS receive antenna, respectively, we can express the overall equivalent channel matrix $\tilde{\mathbf{H}}$ as

$$
\tilde{\mathbf{H}}=\left[\begin{array}{cccc}
\tilde{\mathbf{H}}_{1}^{(1)} & \tilde{\mathbf{H}}_{1}^{(2)} & \ldots & \tilde{\mathbf{H}}_{1}^{(U)} \\
\tilde{\mathbf{H}}_{2}^{(1)} & \tilde{\mathbf{H}}_{2}^{(2)} & \cdots & \tilde{\mathbf{H}}_{2}^{(U)} \\
\vdots & \vdots & \ddots & \vdots \\
\tilde{\mathbf{H}}_{N}^{(1)} & \tilde{\mathbf{H}}_{N}^{(2)} & \cdots & \tilde{\mathbf{H}}_{N}^{(U)}
\end{array}\right] .
$$

Additionally, the transmitted symbol vector $\mathbf{x}$ of the entire MU-MIMO system is a column vector created by concatenating each user's transmitted symbol vector $\mathbf{x}^{(u)}=F_{4}\left(\vec{a}^{(u)}\right)=\left[x_{1}^{(u)} x_{2}^{(u)}\right]^{T}$ which is given by

$$
\begin{aligned}
& \mathbf{x}=\left[\begin{array}{llll}
F_{4}\left(\left(\vec{a}^{(1)}\right)^{T}\right) & F_{4}\left(\left(\vec{a}^{(2)}\right)^{T}\right) & \cdots & F_{4}\left(\left(\vec{a}^{(U)}\right)^{T}\right)
\end{array}\right]^{T}
\end{aligned}
$$

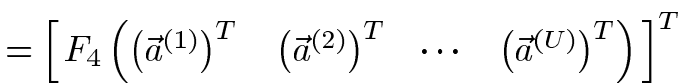

$$
\begin{aligned}
& =\left[\begin{array}{llll}
\left(\mathbf{x}^{(1)}\right)^{T} & \left(\mathbf{x}^{(2)}\right)^{T} & \cdots & \left(\mathbf{x}^{(U)}\right)^{T}
\end{array}\right]^{T} .
\end{aligned}
$$

Thus, by defining $\mathbf{a}=\left[\left(\vec{a}^{(1)}\right)^{T}\left(\vec{a}^{(2)}\right)^{T} \cdots\left(\vec{a}^{(u)}\right)^{T}\right]^{T}$, we have

$$
\mathbf{x}=F_{4}(\mathbf{a}) \text {. }
$$

Moreover, as observed in (3), the equivalent received signal vector $\tilde{\mathbf{y}}$ and the equivalent noise vector $\tilde{\mathbf{w}}$ are formed by concatenating the $N$ two-element sub-vectors $\tilde{\mathbf{y}}_{n}$ and $\tilde{\mathbf{w}}_{n}$, respectively, which can be written as $\tilde{\mathbf{y}}_{n}=\left[\begin{array}{ll}y_{1, n} & y_{2, n}^{*}\end{array}\right]^{T}$, where $y_{1, n}$ and $y_{2, n}$ represent the signals received by the $n$th antenna during the first and second symbol periods, while $\tilde{\mathbf{w}}_{n}=\left[\begin{array}{ll}w_{1, n} & w_{2, n}^{*}\end{array}\right]^{T}$, where $w_{1, n}$ and $w_{2, n}$ denote the AWGN of zero mean and variance $\sigma_{w}^{2}$ inflicted on the $n$th antenna during the first and second symbol periods, respectively.

\section{Sphere Detection Design for SpHere PACKING MOdULATED SYSTEMS}

\section{A. Bit-Based MAP Detection for SP Modulated MU-MIMO Systems}

According to (4) and (9), the conditional PDF $p(\tilde{\mathbf{y}} \mid \mathbf{a})$ for systems using $N_{D}=4$-dimensional real-valued SP modulation is given by

$$
p(\tilde{\mathbf{y}} \mid \mathbf{a})=\frac{1}{\left(2 \pi \sigma_{w}^{2}\right)^{\frac{N_{D}}{2}}} e^{-\frac{1}{2 \sigma_{w}^{2}}\left\|\tilde{\mathbf{y}}-\tilde{\mathbf{H}} \cdot F_{4}(\mathbf{a})\right\|^{2}} .
$$

Then, using the Bayes's theorem, and exploiting the independence of the bits in the vector $\mathbf{b}=\left[b_{1}, b_{2}, \ldots b_{B \cdot U}\right]$ carried by the received symbol vector $\tilde{\mathbf{y}}$, we can factorize the joint bit-probabilities into their products [10]; hence, the LLR of bit $k$ for $k=1, \ldots, B \cdot U$ can be written as

$$
\begin{aligned}
L_{D}\left(\mathbf{b}_{k} \mid \tilde{\mathbf{y}}\right) & =L_{A}\left(\mathbf{b}_{k}\right) \\
+ & \underbrace{\ln \frac{\sum_{\mathbf{a} \in \mathcal{A}_{k=1}^{U}} p(\tilde{\mathbf{y}} \mid \mathbf{a}) \cdot e^{\sum_{j=1, j \neq k}^{B \cdot U} \mathbf{b}_{j} L_{A}\left(\mathbf{b}_{j}\right)}}{\sum_{\mathbf{a} \in \mathcal{A}_{k=0}^{U}} p(\tilde{\mathbf{y}} \mid \mathbf{a}) \cdot e^{\sum_{j=1, j \neq k}^{B \cdot U} \mathbf{b}_{j} L_{A}\left(\mathbf{b}_{j}\right)}}}_{L_{E}\left(\mathbf{b}_{k} \mid \tilde{\mathbf{y}}\right)}
\end{aligned}
$$

where $\mathcal{A}_{k=1}^{U}$ and $\mathcal{A}_{k=0}^{U}$ are subsets of the multiuser SP symbol constellation $\mathcal{A}^{U}$ where we have $\mathcal{A}_{k=1}^{U} \triangleq\left\{\mathbf{a} \in \mathcal{A}^{U}: b_{k}=1\right\}$, and in a similar fashion, $\mathcal{A}_{k=1}^{U} \triangleq\left\{\mathbf{a} \in \mathcal{A}^{U}: b_{k}=0\right\}$. Using (10), we arrive at

$$
\begin{aligned}
& L_{D}\left(\mathbf{b}_{k} \mid \tilde{\mathbf{y}}\right)=L_{A}\left(\mathbf{b}_{k}\right) \\
& +\operatorname{l} \underbrace{\ln \frac{\sum_{\mathbf{a} \in \mathcal{A}_{k=1}^{U}} e^{\left[-\frac{1}{2 \sigma_{w}^{2}}\left\|\tilde{\mathbf{y}}-\tilde{\mathbf{H}} \cdot F_{4}(\mathbf{a})\right\|^{2}+\sum_{j=1, j \neq k}^{B \cdot U} \mathbf{b}_{j} L_{A}\left(\mathbf{b}_{j}\right)\right]}}{\sum_{\mathbf{a} \in \mathcal{A}_{k=0}^{U}} e^{\left[-\frac{1}{2 \sigma_{w}^{2}}\left\|\tilde{\mathbf{y}}-\tilde{\mathbf{H}} \cdot F_{4}(\mathbf{a})\right\|^{2}+\sum_{j=1, j \neq k}^{B \cdot U} \mathbf{b}_{j} L_{A}\left(\mathbf{b}_{j}\right)\right]}}}_{L_{E}\left(\mathbf{b}_{k} \mid \tilde{\mathbf{y}}\right)}
\end{aligned}
$$

\section{B. Sphere Detection Design for Sphere Packing Modulation}

1) Transformation of the ML Metric: The well-known ML solution of (4) is

$$
\hat{\mathbf{a}}_{\mathrm{ML}}=\arg \min _{\check{\mathbf{a}} \in \mathcal{A}^{U}}\left\|\tilde{\mathbf{y}}-\tilde{\mathbf{H}} \cdot F_{4}(\check{\mathbf{a}})\right\|_{2}^{2}
$$

where $F_{4}(\cdot)$ is defined in (9) in the context of our multiuser system. By comparing the unconstrained least-square (LS) solution of $\hat{\mathbf{a}}_{l s}=F_{4}^{-1}\left(\hat{\mathbf{x}}_{l s}\right)=F_{4}^{-1}\left(\left(\tilde{\mathbf{H}}^{H} \tilde{\mathbf{H}}\right) \tilde{\mathbf{H}}^{H} \tilde{\mathbf{y}}\right)$ to all legitimate constrained/sliced solution, namely, à $\in \mathcal{A}^{U}$, the ML solution of (13) can be transformed into

$$
\hat{\mathbf{a}}_{\mathrm{ML}}=\arg \min _{\check{\mathbf{a}} \in \mathcal{A}^{U}} F_{4}\left(\check{\mathbf{a}}-\hat{\mathbf{a}}_{l s}\right)^{H}\left(\tilde{\mathbf{H}}^{H} \tilde{\mathbf{H}}\right) F_{4}\left(\check{\mathbf{a}}-\hat{\mathbf{a}}_{l s}\right) .
$$

2) Channel Matrix Triangularization: Let us now generate the $(2 U \times 2 U)$-dimensional upper-triangular matrix $\mathbf{U}$, which satisfies $\mathbf{U}^{H} \mathbf{U}=\tilde{\mathbf{H}}^{H} \tilde{\mathbf{H}}$ with the aid of Cholesky factorization 
[6]. Then, upon defining a matrix $\mathbf{Q}$ with elements $\mathbf{Q}_{i, i} \triangleq \mathbf{U}_{i, i}^{2}$ and $\mathbf{Q}_{i, j} \triangleq \mathbf{U}_{i, j} / \mathbf{U}_{i, i}$, we can rewrite (14) as

$$
\begin{aligned}
\hat{\mathbf{a}}_{\mathrm{ML}} & \arg \min _{\breve{\mathbf{a}} \in \mathcal{A}^{U}} F_{4}\left(\check{\mathbf{a}}-\hat{\mathbf{a}}_{l s}\right)^{H} \mathbf{U}^{H} \mathbf{U} F_{4}\left(\check{\mathbf{a}}-\hat{\mathbf{a}}_{l s}\right), \\
= & \arg \min _{\breve{\mathbf{a}} \in \mathcal{A}^{U}}\left\{\sum _ { u = 1 } ^ { U } \mathbf { Q } _ { 2 u - 1 , 2 u - 1 } \left[\mathbf{e}_{1}^{(u)}\right.\right. \\
& +\sum_{v=u+1}^{U} \mathbf{Q}_{2 u-1,2 v-1} \mathbf{e}_{1}^{(v)} \\
& \left.+\sum_{v=u}^{U} \mathbf{Q}_{2 u-1,2 v} \mathbf{e}_{2}^{(v)}\right]^{2}+\sum_{u=1}^{U} \mathbf{Q}_{2 u, 2 u}\left[\mathbf{e}_{2}^{(u)}\right. \\
& \left.\left.+\sum_{v=u+1}^{U} \mathbf{Q}_{2 u, 2 v-1} \mathbf{e}_{1}^{(v)}+\sum_{v=u+1}^{U} \mathbf{Q}_{2 u, 2 v} \mathbf{e}_{2}^{(v)}\right]^{2}\right\}
\end{aligned}
$$

where $\mathbf{e}^{(u)}$ is the $u$ th two-element sub-vector of the multiuser vector $\mathbf{e}=\left[\left(\mathbf{e}^{(1)}\right)^{T} \cdots\left(\mathbf{e}^{(u)}\right)^{T} \cdots\left(\mathbf{e}^{(U)}\right)^{T}\right]^{T}$, corresponding to the $u$ th user, and is given by

$$
\mathbf{e}^{(u)}=\check{\mathbf{x}}^{(u)}-\hat{\mathbf{x}}_{l s}^{(u)}
$$

where $\check{\mathbf{x}}^{(u)}=\left[\check{\mathbf{x}}_{1}^{(u)}, \check{\mathbf{x}}_{2}^{(u)}\right]^{T}=F_{4}\left(\vec{a}^{(u)}\right), \vec{a}^{(u)} \in \mathcal{A}$, and $\hat{\mathbf{x}}_{l s}^{(u)}=\left[\hat{\mathbf{x}}_{l s 1}^{(u)}, \hat{\mathbf{x}}_{l s 2}^{(u)}\right]^{T}=F_{4}\left(\hat{\mathbf{a}}_{l s}^{(u)}\right)$. Hence, the sum in $\{\cdot\}$ is the user-based accumulated partial Euclidean distance (PED) between the tentative symbol vector $\check{\mathbf{x}}=\left[\left(\check{\mathbf{x}}^{(1)}\right)^{T},\left(\check{\mathbf{x}}^{(2)}\right)^{T}, \ldots,\left(\check{\mathbf{x}}^{(U)}\right)^{T}\right]^{T}$ and the search center $\hat{\mathbf{x}}_{l s}=\left[\left(\hat{\mathbf{x}}_{l s}^{(1)}\right)^{T},\left(\hat{\mathbf{x}}_{l s}^{(2)}\right)^{T}, \ldots,\left(\hat{\mathbf{x}}_{l s}^{(U)}\right)^{T}\right]^{T}$.

3) User-Based Tree Search: Let us now consider the tree search carried out by the $K$-best SD for conventional modulation schemes, such as BPSK, where each tree level represents an independent data stream corresponding to a certain transmit antenna of a specific user [7], while each tree node corresponds to a legitimate complex-valued BPSK symbol having zero imaginary part in the constellation of domain $\mathbb{C}^{1}$. Hence, in the absence of joint ST signal design for the $M_{u}=2$ transmit antennas, the BPSK constellations of the two adjacent tree levels corresponding to a specific user are independent and identical. Let us now combine the signals transmitted from the $M_{u}=2$ transmit antennas of the $u$ th user into a joint ST design using the SP scheme as discussed in Section II. The corresponding SP-symbol-based search tree of the modified $K$-best SD is depicted in Fig. 2, which is exemplified in the context of a UL SDMA system supporting $U=2 \mathbf{G}_{2}$-SP-aided users, where $K=2$ and the 4-SP scheme is employed. Explicitly, the two adjacent tree levels corresponding to the SP-symbols of the jointly designed STBC-SP data streams of a specific user should be considered together in the tree search process, resulting in 2-D tree nodes in the $\mathbb{C}^{2}$ SP-symbol domain, which we refer to as user-based tree search. Due to the joint consideration of the two adjacent BPSK tree levels, the number of effective search tree levels is reduced by a factor of two, while each symbols becomes quaternary, instead of being binary.

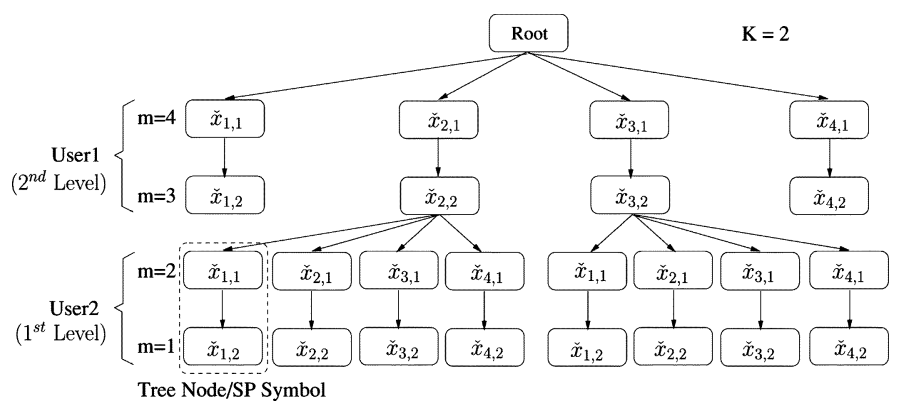

Fig. 2. Search tree of $K$-Best SD in the scenario of an STBC-SP aided uplink SDMA system: the number of users is $U=2$, the number of transmit antennas per user is $M_{u}=2$, and the number of candidates retained at each search tree level is $K=2$.

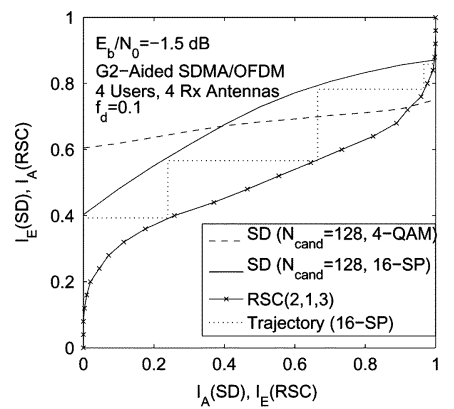

(a)

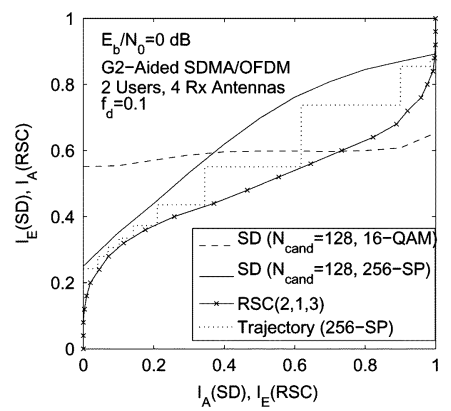

(b)
Fig. 3. EXIT charts of STBC-SP-aided iterative receiver of Fig. 1 employing the modified $K$-best SD and the parameters of Table I. The overall system throughput is 8 bits/symbol. (a) Scenario I. (b) Scenario II.

As observed in Fig. 2, since the 4-SP scheme is employed and the number of candidate tree nodes retained at each tree level is $K=2$, each of the two selected tree nodes having the smallest two accumulated PED values at the previous search-tree level of the survivor path has to be expanded into four child nodes at the current level. Consequently, in analogy to the conventional $K$-best algorithm [7], both the calculation of the user-based accumulated PEDs as well as the tree pruning process continues in the downward direction of Fig. 2, all the way along the tree, until it reaches the tree-leaf level, producing a candidate list of $\mathcal{L}_{\text {cand }} \in \mathcal{A}^{U}$. Having a reduced candidate list size assists us in achieving a substantial complexity reduction in the extrinsic bit LLR calculation. Explicitly, after the max-log-approximation, we arrive at

$$
\begin{aligned}
& L_{E}\left(\mathbf{b}_{k} \mid \tilde{\mathbf{y}}\right) \\
& =\max _{\mathbf{a} \in \mathcal{L}_{\text {cand }} \cap \mathcal{A}_{k=1}^{U}}\left[-\frac{1}{2 \sigma_{w}^{2}}\left\|\tilde{\mathbf{y}}-\tilde{\mathbf{H}} \cdot F_{4}(\mathbf{a})\right\|^{2}\right. \\
& \left.\quad+\sum_{j=1, j \neq k}^{B \cdot U} \mathbf{b}_{j} L_{A}\left(\mathbf{b}_{j}\right)\right] \\
& \quad-\max _{\mathbf{a} \in \mathcal{L}_{\text {cand }} \cap \mathcal{A}_{k=0}^{U}}\left[-\frac{1}{2 \sigma_{w}^{2}}\left\|\tilde{\mathbf{y}}-\tilde{\mathbf{H}} \cdot F_{4}(\mathbf{a})\right\|^{2}\right. \\
& \left.\quad+\sum_{j=1, j \neq k}^{B \cdot U} \mathbf{b}_{j} L_{A}\left(\mathbf{b}_{j}\right)\right] .
\end{aligned}
$$


TABLE I

SUMMARY OF SYSTEM PARAMETERS

\begin{tabular}{|c|c|c|}
\hline & Scenario I & Scenario II \\
\hline Modulation & 4-QAM/16-SP & 16-QAM/256-SP \\
\hline Users Supported & 4 & 2 \\
\hline Doppler Freq. & & 0.1 \\
\hline STBC & & $\mathbf{G}_{2}$ \\
\hline Rx at BS & & $\overline{4}$ \\
\hline Detector/MAP & & $K$-Best List-SD \\
\hline List Length & & $\mathcal{N}_{\text {cand }}=K$ \\
\hline Channel Code & Half-Ra & $\overline{\operatorname{RSC}}(2,1,3)(5 / 7)$ \\
\hline BW Efficiency & & $4 \mathrm{bits} / \mathrm{sec} / \mathrm{Hz}$ \\
\hline
\end{tabular}

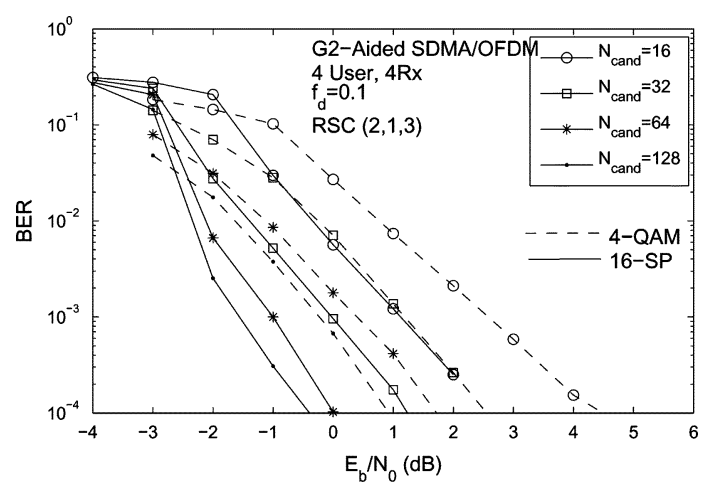

(a)

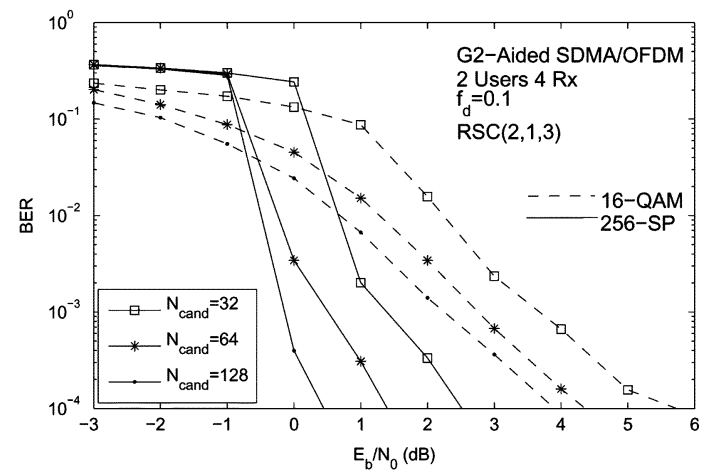

(b)

Fig. 4. BER performance of the system of Fig. 1 in Scenarios $I$ and $I I$ of Table I. The overall system throughput is 8 bits/symbol. (a) Scenario I. (b) Scenario II.

\section{RESULTS AND DISCUSSION}

We compare the SP-modulated system with its conventionally-modulated counterpart in the two scenarios using the system parameters summarized in Table I. Fig. 3(a) and (b) depicts, respectively, the corresponding EXIT charts [11], which computes the mutual information (MI) of the output extrinsic and input a priori components, denoted by $I_{E}$ and $I_{A}$, respectively, corresponding to the associated bits for each of the iterative SISO blocks of Fig. 1. As observed in Fig. 3, the maximum achievable iterative gains of traditional QAM systems employing the conventional $K$-best SD using
$N_{\text {cand }}=K=128$ are rather limited in comparison to our $K$-best SD specifically designed for SP signals having the same list size. This is because the EXIT curve of the SD used by the conventional 4- and 16-QAM-based system has a relatively low $I_{E}$ value at $I_{A}=1$, in contrast to the corresponding EXIT curve of its SP-modulated counterpart. Nonetheless, we also observe from Fig. 3 that the SD's EXIT curve in the QAM-aided system emerges from a higher starting point at $I_{A}=0$ than that of its SP-modulated counterpart. This leads to a potentially lower BER at relatively low SNRs, where $I_{A}$ is also low, although the exact detection-convergence behavior is determined by the SD's complexity as well as by the SNR. Observe in Fig. 3 that in principle, the employment of SP modulation is capable of eliminating the EXIT curve intercept point at a lower SNR, hence leading to an infinitesimally low BER. However, an open EXIT tunnel can only be formed, if the value of $K=N_{\text {cand }}$ as well as that of the SNR is sufficiently high.

Monte Carlo simulations were performed for characterizing the above-mentioned decoding convergence prediction in both Scenarios I and II of Table I. Fig. 4(a) and (b) suggests that the SP-modulated system exhibits a relatively higher BER at low SNRs in both scenarios, which matches the predictions of the EXIT charts seen in Fig. 3. On the other hand, as a benefit of employing the SP modulation, performance gains of $1.5 \mathrm{~dB}$ and $3.5 \mathrm{~dB}$ can be achieved by $16-\mathrm{SP}$ and $256-\mathrm{SP}$ modulated systems in Scenarios I and II of Table I, respectively, in comparison to their identical-throughput QAM-based counterparts, given a target BER of $10^{-4}$ and $N_{\text {cand }}=128$.

\section{REFERENCES}

[1] J. H. Conway and N. J. A. Sloane, Sphere Packings, Lattices and Groups, 3rd ed. New York: Springer-Verlag, 1999.

[2] W. Su, Z. Safar, and K. J. R. Liu, "Space-time signal design for timecorrelated Rayleigh fading channels," in Proc. IEEE Int. Conf. Communications 2003, May 2003, vol. 5, pp. 3175-3179.

[3] S. M. Alamouti, "A simple transmit diversity technique for wireless communications," IEEE J. Select. Areas Commun., vol. 16, no. 8, pp. 1451-1458, Oct. 1998.

[4] V. Tarokh, H. Jafarkhani, and A. R. Calderbank, "Space-time block codes from orthogonal designs," IEEE Trans. Inf. Theory, vol. 45, no. 5, pp. 1456-1467, Jul. 1999.

[5] W. Su and X. G. Xia, "On space-time block codes from complex orthogonal designs," Wirel. Pers. Commun., vol. 25, pp. 1-26, Apr. 2003.

[6] E. Viterbo and J. Boutros, "A universal lattice code decoder for fading channels," IEEE Trans. Inf. Theory, vol. 45, no. 5, pp. 1639-1642, Jul. 1999.

[7] Z. Guo and P. Nilsson, "Algorithm and implementation of the K-best sphere decoding for MIMO detection," IEEE J. Select. Areas Commun., vol. 24, no. 3, pp. 491-503, Mar. 2006.

[8] L. Hanzo, M. Munster, B. J. Choi, and T. Keller, OFDM and MC-CDMA for Broadband Multi-User Communications, WLANs and Broadcasting.. Piscataway, NJ: IEEE Press, 2003.

[9] L. Hanzo, T. H. Liew, and B. L. Yeap, Turbo Coding, Turbo Equalisation and Space-Time Coding for Transmission over Fading Channels.. Piscataway, NJ: IEEE Press, 2002.

[10] B. M. Hochwald and S. ten Brink, "Achieving near-capacity on a multiple-antenna channel," IEEE Trans. Commun., vol. 51, no. 3, pp. 389-399, Mar. 2003.

[11] S. ten Brink, "Convergence behavior of iteratively decoded parallel concatenated codes," IEEE Trans. Commun., vol. 49, no. 10, pp. 1727-1737, Oct. 2001. 